\section{Cahiers de Narratologie}

Analyse et théorie narratives

$19 \mid 2010$

Images composites, arts pluriels

\title{
Diversité esthétique et pluralité des images - un exemple francophone
}

Noémi Kila

\section{(2) OpenEdition}

Journals

Édition électronique

URL : http://journals.openedition.org/narratologie/6208

DOI : 10.4000/narratologie.6208

ISSN : 1765-307X

Éditeur

LIRCES

Référence électronique

Noémi Kila, "Diversité esthétique et pluralité des images - un exemple francophone », Cahiers de Narratologie [En ligne], 19 | 2010, mis en ligne le 22 décembre 2010, consulté le 19 avril 2019. URL http://journals.openedition.org/narratologie/6208; DOI : 10.4000/narratologie.6208

Ce document a été généré automatiquement le 19 avril 2019

\section{(c) $($ i) $(9)$}

Cahiers de Narratologie - Analyse et théorie narratives est mis à disposition selon les termes de la licence Creative Commons Attribution - Pas d'Utilisation Commerciale - Pas de Modification 4.0 International. 


\title{
Diversité esthétique et pluralité des images - un exemple francophone
}

\author{
Noémi Kila
}

1 Le croisement des différents registres langagiers et des différents domaines de l'art est depuis toujours considéré comme particulièrement productif et créateur d'esthétiques "hors cadre». Quand vient s'y ajouter le dynamisme langagier et esthétique caractérisant les littératures francophones, la «surconsience linguistique » et l'«esthétique du divers" - dans le sens auquel Lise Gauvin ${ }^{1}$ et Victor Segalen ${ }^{2}$ les entendent - se manifestent d'une manière plus prononcée. La problématique de la langue est bien explicitée par les propos d'Étienne Barilier : «Je suis français : voilà qui unit, dans les profondeurs de la sémantique, langue et nation. "Je suis un Suisse d'expression française" : voilà qui définit tant bien que mal et voilà qui disjoint. Comment, dans ces conditions, ne pas en venir à penser que toute langue est relative, toute culture contingente ${ }^{3}$ ?»

2 Les notions de «transgression » et d'« hybridation » caractérisent plus particulièrement les œuvres francophones. «Faut-il lire dans ces pratiques de décentrement, de rupture, voire de transgression et de questionnement du statut des langues et de la littérature des exemples de post-modernisme? Assurément, puisque la plupart de ces poétiques, fondées sur l'hétérogénéité, problématisent la notion même de la langue et du langage ${ }^{4}$ »constate Gauvin. En ce qui concerne les pratiques langagières d'hybridation et d'impureté, elle les associe à la dédramatisation des tensions linguistiques et parle de la «variance infinie de nuances poétiques possibles des langues 5 .» Les procédés analogues aux pratiques langagières présents dans les corpus littéraires francophones se laissent également découvrir au niveau des démarches esthétiques et narratives exploitées dans les œuvres en question. 


\section{Réflexion sur une écriture d'entre-deux}

3 Les textes de l'auteur suisse romand Charles-Ferdinand Ramuz s'inscrivent dans l'idée du croisement des registres langagiers, ainsi que dans celle du carrefour de l'esthétique du visible et du lisible. Ces caractéristiques se manifestent principalement par la remise en cause des normes langagières et la dimension transgressive des choix esthétiques relatifs aux différentes représentations de l'image. L'auteur oppose la notion de la «languesigne » à celle de la "langue-image ${ }^{6} »$. Pour lui, si l'on veut comprendre le monde, on a besoin de le percevoir à travers les images: "Le sens surgit - pour le monde et pour moi - dans la réciprocité fondatrice de l'image-perception ${ }^{7}$.» La réciprocité que Ramuz considère inhérente à l'« image-perception " présuppose un mouvement continu entre image et perception. Cette dernière fonctionne comme créatrice d'images apparaissant dans une pluralité impressionnante, allant de pair avec une diversité esthétique aux frontières du littéraire et du cinématographique. L'univers des protagonistes ainsi que celui du monde romanesque sont notamment construits comme des suites de fragments du monde perceptible, analogue à une juxtaposition d'images, mais s'éloignant d'une unité quelconque. Cinéma et littérature se nouent et constituent un univers où se met en place une véritable "langue-image». La juxtaposition des images rend possible la projection des fragments du monde perçu. Ramuz évoque l'importance des « morceaux » dans son Journal : « [J]e n'obéis qu'à l'impression; je vois quelque chose avec intensité, je le fixe. Mais ce quelque chose n'est lié en rien à cette autre chose qui suit: c'est un morceau ; j'y juxtapose un second morceau; ce sont les touts qui ne font pas un tout $^{8}$." L'auteur voit le tout non pas dans une unité construite à partir d'éléments juxtaposés, mais dans des morceaux fixés par la vue, des miettes prises, filmées par le regard et enregistrées par l'ouie.Il voit notamment l'essence du roman dans l'invention qui "est dans la vue éclatante ; elle est dans l'image ; elle est dans le mouvement de la phrase ; elle n'est pas ailleurs9. " L'image se présente donc-dans l'esthétique ramuzienne - comme un élément esthétique de base, toujours en rapport avec la perception et la langue, comme le montrent les conjonctions « langue-image » et « langue-perception ».

Selon notre hypothèse, la démarche ramuzienne des années 1910-1930 crée une esthétique de l'entre-deux, aux limites du littéraire et du cinématographique. Afin de montrer la pertinence de cette hypothèse, on propose d'abord un tour d'horizon de l'univers de la critique littéraire, notamment trois approches classiques de l'œuvre en question, qui nous permettra de les nuancer et de nous positionner du côté de la diversité esthétique. Ensuite, nous nous consacrons à l'étude des différents aspects de l'esthétique en question pour enfin proposer une piste de réflexion sur une nouvelle hypothèse selon laquelle l'esthétique forgée par Ramuz dans les années 1910-1930 et celle des nouveauxromanciers des années 1950-1970 pourraient être examinées dans une perspective comparative, non seulement au niveau de la prédominance des effets.cinématographiques, mais aussi à celui de leur caractère discontinu et divers.

Pour dresser l'image des différentes positions critiques parues sur l'écriture ramuzienne, nous prenons en considération les différentes époques (celle de la parution des premiers romans de Ramuz, celle des années soixante - époque du renouveau critique qui s'est fait autour de l'œuvre ramuzienne - et l'époque contemporaine) et les différents pays où des études sur la production romanesque de l'auteur suisse romand ont vu le jour (tels la 
France, la Suisse et le Québec). Premièrement, à l'époque de leur parution, les premiers textes de Ramuz ont souvent été jugés incorrects par rapport aux normes grammaticales et syntaxiques imposées par l'Académie Française ou réduite à la reproduction de l'oralité. Cette attitude caractérisait non seulement les critiques françaises, mais également certaines critiques suisses. En ce qui concerne les critiques françaises, deux cas types se dessinent. Le premier est bien représenté par la critique d'Auguste Bailly : «Écrivain français!... S'il veut l'être, qu'il apprenne notre langue!... Et s'il ne veut pas l'apprendre, qu'il en emploie une autre ${ }^{10}$ ! . L'autre cas type se résume dans les reproches de Kemp, publiés dans la Revue Contemporaine: "Langage fort fatigant [...], à force d'afféterie et de truquage. Mépris absolu de la syntaxe, absence complète de concordance des temps, oubli volontaire des verbes, emploi des vocables du pur patois vaudois ${ }^{11}$. » En ce qui concerne les critiques suisses, l'une - parue en 1911 -affirme que le langage ramuzien est loin d'être du français: « Ceci [...] n'est pas français : Il y avait un reste de vin rouge dans une bouteille ; il était acide et âpre à la fois ; il en but trois à quatre verres ${ }^{12}$. " La deuxième approche - celle de Parsons publiée au Québec - s'alimente essentiellement de la biographie et du Journal de l'auteur. Pour prouver que Ramuz souhaite créer une vision plastique, Parsons s'appuie sur des extraits du Journal de l'auteur et sur l'étude de ses rapports avec des peintres. « Ramuz va essayer de nous exprimer sa vision plastique de son pays et de ses habitants ${ }^{13}$.»-remarque-t-il. Son étude démontre en quoi l'art ramuzien peut être rapproché de celui des impressionistes et cubistes, mais tente également de définir dans quelle mesure la relation particulière de Ramuz à la peinture a $\mathrm{pu}$ influencer son style et sa langue. Parsons insiste aussi sur le vocabulaire pictural présent dans l'œuvre ramuzienne et attire notre attention sur la prédominance des verbes suivants : avoir, être, faire, montrer, regarder, voir ${ }^{14}$. La troisième approche, celle de Villelm milite d'une part pour « l'ambiguïté du narrateur ${ }^{15}$ » et insiste d'autre part sur sa "fonction limitée ${ }^{16}$." Elle plaide notamment pour une "voix naïve du narrateur ramuzien ${ }^{17}$ » et désigne la perspective narrative en question comme «essentiellement actorielle ${ }^{18} »$. D'autre part, Villelm met en avant le fait que « le monde matériel est décrit chez Ramuz d'après les impressions des personnages ${ }^{19}$." En 2001, le même auteur considère que les romans ramuziens parus entre 1926 et 1937 sont basés sur une dramaturgie implicite et conclut ainsi :

[L]'espace ramuzien, comme l'espace scénique, est un espace clos et souvent représenté comme factice. Ce rapport à l'espace scénique apparaît en premier lieu dans la clôture de l'espace. Le cadre montagnard est un univers hermétique dans lequel existent encore nombre d'espaces de la clôture, lieux de scènes dialoguées et gestualisées ${ }^{20}$.

6 En soulignant l'importance des dialogues et l'abondance des espaces clos dans les romans ramuziens, Villelm nous reconduit en effet aux idées suivantes: la reproduction de l'oralité et la représentation des espaces typiques de la Suisse.

$7 \quad$ Les approches présentées sont récusables pour différentes raisons. Le point faible de la première approche - accusant Ramuz de ne pas être français - est de caractère plutôt sociologique que littéraire. De plus, la présence des fautes grammaticales et syntaxiques n'exclut pas qu'il s'agisse d'un langage riche et inventif qui - de temps en temps - tire même profit de ses gaucheries. Comme l'a suggéré Starobinski dans un article consacré à Ramuz, il faudrait convertir l'écart par rapport aux normes académiques en plus-value artistique : "Sans doute y a-t-il à l'origine une certaine défiance envers le langage. Mais cette défiance, cette gêne, qui sont imposées à tant d'hommes de l'extrême périphérie du domaine linguistique français, il faudra savoir leur faire jouer un rôle salutaire ${ }^{21}$.» Il faut 
également remarquer que - pour ne commenter qu'un exemple des reproches formulés par Kemp - le soi-disant " oubli volontaire des verbes » se transforme - après une analyse plus approfondie - en style nominal au service d'une esthétique scénaristique proche de l'esthétique robbe-grilletienne. Ensuite, les deux autres approches présentées ont comme point de départ la biographie de l'écrivain, son Journal ou son lieu natal. Quoique Parsons s'appuie sur des éléments biographiques, son étude propose également une tentative d'analyse du langage ramuzien. En ce qui concerne l'approche de Villelm (2001), notons qu'à la fin de sa réflexion, elle remet en question la comparaison effectuée entre les romans de Ramuz et les pièces de théâtre. L'auteur dédie notamment un sous-chapitre à la présentation de quelques effets cinématographiques, sans exhaustivitée ${ }^{2}$. La fragilité de sa position critique réside entre autres dans son constat suivant: "Ramuz célèbre l'espace vaste, l'immensité de la montagne en usant et abusant de techniques empruntées au septième $\operatorname{art}^{23}$. Ce constat a recours au lieu natal de l'auteur et réduit l'importance des techniques cinématographiques à la représentation de l'espace et plus précisément à celle de la montagne. Or, la présence des aspects cinématographiques est beaucoup plus forte et ne se réduit pas à la visualisation de l'espace. En somme, nous constatons que pour une analyse approfondie des particularités de l'écriture en question - au lieu de s'appuyer sur la biographie de l'écrivain ou sur ses rapports aux arts -, il s'avère plus efficace de se pencher sur l'étude des stratégies d'expression et techniques narratives présentes dans l'œuvre de l'auteur suisse romand. Celle-ci permet notamment de rendre compte d'une esthétique originale qui met en avant l'image sous toutes ses formes.

\section{Une instance narrative « hors-cadre »}

8 L'écriture ramuzienne basée sur une juxtaposition d'images, se caractérise par une narration particulière, par une instance narrative apparaissant le plus souvent sous la forme grammaticale du pronom « on", non-identifiable et relevant du caractère nonpersonne. Maingueneau explique la polyvalence du «on» de la façon suivante: sa référence varie selon le contexte énonciatif et peut renvoyer à la «non-personne ${ }^{24}$ ». Aussi, le même auteur constate que le « on » " subvertit l'opposition entre " personne » et «non-personne ${ }^{25} »$. Deleuze consacre également une réflexion à la troisième personne : «Mais la littérature (...) ne se pose qu'en découvrant sous les apparentes personnes la puissance d'un impersonnel qui n'est nullement une généralité mais une singularité au plus haut point (...). Ce ne sont pas les deux premières personnes qui servent de condition à l'énonciation littéraire; la littérature ne commence que lorsque naît en nous une troisième personne qui nous dessaisit du pouvoir de dire Je (le "neutre de Blanchot $^{26 ")}$.» Ce trait est susceptible d'être pertinent pour le rapprochement entre l'écriture en question et les techniques cinématographiques. L'alternance entre perception et image, ainsi qu'une apparente objectivité, relevant du caractère anonyme, non-identifié du narrateur peut être observé dans l'exemple suivant:

Séraphin a levé le bras. On voit sa main dans la nuit claire. Séraphin montre là-haut quelque chose (...). Séraphin lève le bras, il fait naître devant vous un nouveau mur, plus haut que tous les autres; cependant que ce grand mur est tout creusé d'étroites gorges où pendent en bougeant de petites cascades. Le regard le parcourt lui aussi de bas en haut; puis il y a le doigt tendu de Séraphin qui oblige l'œil à s'arrêter ${ }^{27}$.

Dans cet extrait, on observe l'apparition de l'expression « on voit»: ce «on » pourrait avoir différentes interprétations dans ce contexte. Soit, il s'agirait d'un « on » personnel, 
soit d'un « on » impersonnel. Plus loin, il y a des indices qui permettent de lui attribuer le caractère impersonnel : il s'agit notamment du « regard » et de « l'œil » anonymes de la dernière phrase. Étant donné que le contexte ne représente pas d'autres protagonistes à qui il serait possible d'attribuer le processus de perception visuelle, nous sommes contraints d'interpréter le « on » en question comme relevant de la catégorie linguistique impersonnelle. De plus, il rappelle les mouvements de la caméra dans la mesure où tout d'abord, il fixe la main du personnage, puis balaye le mur pour enfin s'arrêter sur l'image initiale. Le caractère mécanique de ce type de narrateur ressort dans le processus de la reproduction des mouvements de la caméra sans qu'il s'agisse d'un point de vue tout à fait objectif.

Afin de mieux éclairer le fonctionnement de l'instance narrative ramuzienne, voici un autre exemple pour l'œil mécanique présent dans l'extrait suivant :

Oh! il est beaucoup trop petit.

A cinq cents mètres au-dessous de vous, il ne serait qu'un minuscule point blanc, non perceptible à l'œil nu parmi l'immensité de ces déserts ${ }^{28}$.

11 Dans ce cas, on assiste à la description du processus d'élévation du narrateur en dessus du personnage qui lui apparaîtrait comme "un minuscule point blanc». La proposition «non perceptible à l'œil nu » sous-entend perceptible seulement à un œil autre que nu, c'est-à-dire qui est capable de voir plus qu'un œil humain. L'œil de la caméra correspond parfaitement à cette exigence. Le conditionnel «il ne serait que » rappelle le caractère purement hypothétique de l'image perçue par l'œil fixant le personnage en question. On assiste donc soit à une prise de vue par un narrateur mécanique, soit à une prise de vue hypothétique.

Pour clore cette réflexion sur le caractère «non-personne » et "non-identifié » de l'instance narrative, on a recours à une idée formulée par Philippe Renaud qui considère que la pratique d'écriture ramuzienne et l'art d'« écrire (...) que quelque chose "fut vu ", sans direqui l'a vue ${ }^{29} . » \mathrm{La}$ "fonction limitée » du narrateur dont parlait Villelm ne se défend donc pas. La non-identification du narrateur, les zones d'indétermination constituées par l'instance narrative créent une narration d'entre-deux, une esthétique de possibles narratifs et poétiques.

Il s'agit non seulement des facteurs d'indétermination comme éléments créateurs de la diversité esthétique; la pluralité des images, notamment analogues aux images cinématographiques viennent les compléter et enrichissent ainsi cette narration toujours « à cheval ».

\section{Pluralité des images et échos cinématographiques}

Les images surgissant dans la narration ramuzienne doivent leur statut pluriel d'une part aux différentes formes sous lesquelles elles apparaissent, d'autre part à la présence de différentes perspectives qui s'y entremêlent. Du point de vue esthétique, elles se situent à la limite du littéraire et du cinématographique.

15 La scène où les protagonistes sont assis autour du feu assure un cadre permettant l'exploitation des dispositions offertes par le procédé cinématographique du balayage :

À côté du maître, il y avait le neveu, puis on passait à Joseph, puis à Barthélémy. Barthélémy faisait face à la paroi (...). Il y avait donc cette paroi ; il y avait, en face de la paroi la figure de Barthélémy (...), une bouche qu'on ne voyait pas (et on n'en devinait la place qu’à la direction que prenait le tuyau de la pipe) (...). Barthélémy 
faisait face à la paroi (...), entre Joseph et Romain, puis venaient à sa droite Clou et le boûbe, et le maître et le neveu du maître étaient à sa gauche. Barthélémy était assis face à la paroi ; et, quand on regardait Barthélémy, on voyait que sa grosse barbe bougeait toujours, mais il ne disait rien ${ }^{30}$.

Le regard se pose d'abord sur le maître, puis sur le neveu et ensuite sur Joseph pour enfin s'arrêter sur Barthélémy. Le« on" de "on passait» de cette scène renvoie au onnarrateur qui explore avec son regard les personnages du roman. De plus, le « on » de "quand on regardait Barthélémy, on voyait que sa grosse barbe bougeait » permet de définir qu'il s'agit d'une focalisation sur Barthélémy. Ce passage en revue des personnages et l'arrêt de l'œil du narrateur sur Barthélémy préparent aussi une histoire à raconter, notamment une ancienne histoire de la montagne que racontera Barthélémy lui-même. La focalisation sur un personnage dans le but de préparer son discours est un élément constitutif de la cinématographie. La perspective du narrateur balayant du regard les protagonistes assis en cercle est mise en évidence par la structure « il y avait » et les occurrences $d u$ "on» des trois premières phrases auxquelles s'ajoute le «on » intercalé entre les parenthèses : vu que cette occurrence du pronom est associée à un verbe présupposant la cognition humaine («deviner») et permettant ainsi l'émergence d'une perspective humaine, voire celle d'un personnage, l'image cinématographique se trouve entrecoupée pour un instant, afin de se clore sur une focalisation de type cinématographique marquée par l'alternance des verbes « regarder » et « voir ».

L'image en mouvement est également présente dans la narration ramuzienne. Sa visualisation concorde avec celle de l'image rapide cinématographique et fait appel - cette fois-ci - à la perspective du narrataire :

La côté de Savoie, dans cet instant, s'est découverte.

Pour un instant seulement, on l'a eue de nouveau devant soi ; elle s'est défaite de ses vapeurs : elle brille singulièrement, elle est toute peinte en vert clair; on dirait qu'on va la toucher, tellement elle s'est rapprochée.

Elle vient à vous comme sur des roues; elle vient à vous comme une barque sous ses voiles avec ses grandes montagnes qui semblent toutes gonflées de vent; elle se rapproche encore, arrêtez!

Et elle est là, pour un instant, avec ses forêts, ses prés, ses villages, ses champs, ses vignes, ses rochers, - tout ça se marque dans le plus grand détail, par lignes, traits, contours, rectangles, taches rondes, sur son coloriage comme sur une carte de géographie ; et il nous est dit : «Regardez! $»^{31}$.

Ce sont les verbes de mouvement, le temps du présent et l'insantanéité qui dominent ce passage. Le syntagme «Elle vient à vous comme sur des roues » évoque la caméra qu'on roule sur un praticable. L'image des roues est tout de suite remplacée par celle des voiles impliquant aussi la vitesse. L'impératif « arrêtez!» indique que l'image est susceptible d'écraser le spectateur-narrataire. Bien que dans les récits ramuziens, le narrateur apparaisse souvent en tant qu'impersonnel et mécanique - comme pourrait suggérer le passif « il nous est dit »-il ne reste pas tout à fait objectif puisqu'il se raccorde en même temps avec les énoncés « arrêtez ! » et «Regardez ! ». Étant donné que le texte ne fournit aucun indice pour identifier l'interlocuteur de ces deux énoncés, ils sont susceptibles d'être adressés aux personnages et au narrataire en même temps qui devra ainsi participer à la construction de la logique narrative. Comme le rappelle Leiris : « [l]'œuvre doit être lisible, mais non sans un certain effort qui nous mobilise, elle requiert de notre part une participation active $\mathrm{e}^{32}$.» 


\section{Superposition des images et discontinuité des perspectives}

Les processus liés à l'accumulation des images et à la discontinuité sont également présents dans l'arrangement et la structure-même des images ramuziennes. La juxtaposition des images caractérisant la narration ramuzienne se transforme par endroit en superposition des images. Quant à la discontinuité, elle caractérise également nombre d'images ramuziennes et prend la forme de la discontinuité des perspectives. Ces deux cas spécifiques correspondent à deux conceptions originales de l'image en littérature.

L'exemple de l'image qui n'est là que pour ensuite être effacée ou plutôt mélangée avec une autre représente la « superposition des images »:

Et cette verrerie est située à un bon demi-kilomètre du village; pourtant, distinctement, ces coups de trompe se font entendre jusqu'au village, - il y en a quatre là-bas qui sont tombés devant les fours. (...) Tout le jour, toute la nuit ces fours à verre sont allumés; tout le jour, toute la nuit (le jour en blanc, la nuit en rouge), on les voit luire sous les hangars; on ne les voit pas seulement.

Car il y a ceux qui sont devant. Ils prennent un peu de verre au bout de la canne. (...) Ils chantent. On voit ces fours rouges et blancs; eux sont devant, on les entend ${ }^{33}$.

1 Dans cet extrait, le bruit intervient en tant qu'élément formant le cadre de la scène : d'une part, le bruit des trompes sert d'introduction, d'autre part, le passage se clôt sur le chant des hommes qui se trouvent devant les fours. L'image des fours se dresse devant nos yeux et ce n'est que par la négation commençant avec le deuxième « on » et le nouvel alinéa "Car il y a ceux qui sont devant », que l'image initiale se trouve bouleversée et ne réapparaît que dans la dernière phrase de l'extrait soulignant la non-vue des fours. La même impression esthétique est présente au cinéma où l'image suit l'image ; le mélange des effets sonores et visuels de sa part ne fait que renforcer l'ancrage cinématographique du texte.

Le caractère discontinu des perspectives a déjà été souligné par Christian Morzewski dans son analyse consacrée aux nouvelles ramuziennes. Morzewski insiste notamment sur la « $[\mathrm{m}]$ obilité et versatilité des points de vue adoptés » et de l' « indicibilité ultime du foyer principal de description ${ }^{34}$. » Dans ce dernier exemple de l'esthétique ramuzienne, il s'agit de la suite discontinue de deux, voir trois perspectives différentes qui surgissent dans un contexte de quelques lignes subséquantes :

Il y avait (...) cette réunion de petits toits qui se tenaient serrés sous leurs petites fumées bleues. A travers la couleur de ces fumées, on voyait la couleur des ardoises, ces murs faits en vieilles poutres. (...) On voyait que les toits se tenaient là (...) - et Clou disait que ça ne pressait pas ; - on voyait aussi (...) les jardins (...). Victorine et Joseph étaient derrière la haie, ils s'y trouvaient à l'abri des regards. Il y avait en face d'eux, les montagnes qui devenaient roses ${ }^{35}$.

La discontinuité des points de vue se présente de la manière suivante : vu qu'il n'y a pas d'indices spéciaux permettant l'identification du premier «on», il peut aussi bien renvoyer au couple désigné dans la dernière phrase qu'au narrateur. La coprésence du formule «il y avait » et du pronom «on ", déjà évoquée lors de l'étude des échos cinématographiques, est également à souligner. Le troisième point de vue est celui de Clou, un personnage qui ne se trouve pas dans la même scène mais dont la perspective s'intercale entre les deux perspectives voisines. L'image complète se construit donc des trois points de vue voisinant dans le texte, mais incarnant trois positions narratives 
différentes: celle d'une instance narrative non-identifiée qui voit les murs et les toits d'une certaine distance et d'une certaine hauteur ; celle du personnage Clou dont le point de vue vient entrecouper la perception associée à l'instance " on »; et celle du couple (Joseph et Victorine) dont la position est bien définie. La discontinuité des différentes prises de vue est créatrice d'une conception de rassemblage produisant une image composite.

\section{Conclusion}

La tension entre les parties et le tout de la narration ramuzienne se manifeste bien dans l'écart qui sépare la thématique des textes en question de leur composition esthétique. L'étude entreprise dans le cadre de cet article nous a permis de remettre en question les approches classiques de l'œuvre de l'auteur suisse romand et de la placer dans une perspective à la fois esthétique et narrative. La singularité de l'instance narrative - dont l'identification demande souvent un travail de déchiffrement -, le caractère pluriel des textes se manifestant sous forme de mélange du littéraire et du cinématographique, ainsi que la juxta- et superposition des images et la discontinuité des perspectives sont des éléments qui confirment qu'il s'agit d'une esthétique éminemment moderne, basée sur les différentes formes d'hybridité, de diversité et de possibles poétiques. Une étude sur les éventuelles analogies entre la technique d'écriture ramuzienne et celle du roman cinéoptique des années 1920, ainsi que celle du nouveau roman des années 1950-1960 serait particulièrement enrichissante dans la mesure où les différentes formes de l'image et de l'illusion cinématographique, l'ambiguïté référentielle et le statut du narrateur sont des éléments dominants dans ces trois types de texte et sont créateurs d'esthétiques originales.

\section{BIBLIOGRAPHIE}

Deleuze, Gilles : Critique et clinique, Paris, Minuit, 1993.

Gauvin, Lise : L'écrivain francophone à la croisée des langues - Entretiens, Paris, Karthala, 1997.

Helkulla, Mervi : « Énonciation et subjectivité dans La Première Gorgée de bière et autres plaisirs minuscules ».Dans : Poétique, $\mathrm{n}^{\circ}$ 147, 37 année, septembre 2006, pp. 317-325.

Maingueneau, Dominique : Éléments de linguistique pour le texte littéraire, Paris, Dunod, 1993.

Meizoz, Jérôme : « Le droit de "mal écrire" - Trois cas helvétiques (XVIII - XX ${ }^{\mathrm{e}}$ siècle) ».Dans : Actes de la recherche en sciences sociales, Paris, Seuil, $\mathrm{n}^{\circ}$ 111-112, mars 1996, pp. 92-109.

Morzewski, Christian : «Regard et point de vue dans les nouvelles de Ramuz - essai d'optique ramuzienne. » Dans : Ramuz et la forme brève. (Textes présentés et réunis par Jean-Louis Pierre), La Revue des Lettres Modernes, Paris, Caen, 2003, pp. 173-194.

Nagyné Schmelzer, Erika : « Le journal de C.-F. Ramuz : à la recherche d'un style ».Dans : Őrsi Tibor (réd.) : Acta academiae Paedagogicae Agriensis, Tome XXX., Eger, 2003, pp. 119-124. 
Parsons, Clarence R. : Vision plastique de C.-F. Ramuz, Les Presses de l'Université de Laval, Québec, 1964.

Péguy, Marcel : Pour ou contre Ramuz, Cahier de témoignages, Paris, Editions du Siècle, collection Cahiers de la Quinzaine, 1926.

Ramuz, Charles-Ferdinand : Derborence. Dans : Cuvres complètes, vol. 17, Genève, Slatkine, 1986, pp. 175-347.

Ramuz, Charles-Ferdinand : Derborence, Paris, Bernard Grasset, 1936.

Ramuz, Charles-Ferdinand : La grande peur dans la montagne. Dans : Euvres complètes, vol. 13, Genève, Slatkine, 1986, pp. 149-323.

Ramuz, Charles-Ferdinand : Les signes parmis nous. Dans : Cuvres complètes, vol. 10, Genève, Slatkine, 1986, pp. 45-172.

Ramuz, Charles-Ferdinand : Romans, Paris, Gallimard, collection Bibliothèque de la Pléiade, 2005. Renaud, Philippe : Ramuz ou l'intensité d'en bas, Lausanne, L'aire critique, 1986.

Rossel, Virgile : Article paru le 4 juin 1911 dans Le National Suisse. (Article conservé dans le Centre de Recherches sur les Lettres Romandes à Lausanne.)

Segalen, Victor : Essai sur l'exostime : une esthétique du divers, Paris, Fata Morgana, 2007.

Skander, Kamel : «La discontinuité, une esthétique de l'écriture chez Leiris. » Dans : Poétiques de la discontinuité de 1870 à nos jours (Études rassemblées et présentées par Isabelle Chol), ClermontFerrand, Presses Universitaires Blaise Pascal, 2004, pp. 107-115.

Villelm, Sylvie : La dramaturgie implicite dans les romans de Charles-Ferdinand Ramuz (1926-1937). Thèse de doctorat, Université Aix-Marseille I - Université de Provence, 2001.

Villelm, Sylvie : La forme ramuzienne : une écriture de l'implicite, Université Aix-Marseille I Université de Provence, DEA, 1995.

\section{NOTES}

1. Voir: Lise Gauvin, L'écrivain francophone à la croisée des langues - Entretiens, Paris, Karthala, 1997, pp. 9-10.

2. Voir : Victor Segalen, Essai sur l'exotisme : une esthétique du divers, Paris, Fata Morgana, 2007.

3. Cité par Lise Gauvin, op. cit., p. 8.

4. Idem., p. 14.

5. Ibid.

6. Philippe Renaud, Ramuz ou l'intensité d'en bas, Lausanne, L'aire critique, 1986, p. 23.

7. Idem, p. 20.

8. Cité par Nagyné Schmelzer Erika : «Le journal de C.-F. Ramuz: à la recherche d'un style». Dans : Őrsi Tibor (réd.) : Acta academiae Paedagogicae Agriensis, Tome XXX., Eger, 2003, p. 121.

9. Charles-Ferdinand Ramuz, Romans, Paris, Gallimard, collection Bibliothèque de la Pléiade, 2005, p. XII.

10. Marcel Péguy, Pour ou contre Ramuz, Cahier de témoignages, Paris, Editions du Siècle, collection Cahiers de la Quinzaine, 1926, p. 241.

11. Idem, p. 256.

12. Voir l'article de Virgile Rossel paru le 4 juin 1911 dans Le National Suisse. (Article conservé dans le Centre de Recherches sur les Lettres Romandes à Lausanne.) 
13. Clarence R. Parsons, Vision plastique de C.-F. Ramuz, Les Presses de l'Université de Laval, Québec, 1964, p. 18.

14. Idem, p. 26.

15. Sylvie Villelm : La forme ramuzienne: une écriture de l'implicite, Université Aix-Marseille I Université de Provence, DEA, 1995, p. 7-11.

16. Idem, p. 21.

17. Idem, p. 28.

18. Idem, p. 29.

19. Idem, p. 87.

20. Sylvie Villelm, La dramaturgie implicite dans les romans de Charles-Ferdinand Ramuz (1926-1937). Thèse de doctorat, Université Aix-Marseille I - Université de Provence, 2001, p. 384.

21. Cité par Jérôme Meizoz, "Le droit de "mal écrire" - Trois cas helvétiques (XVIIIe-XXe siècle) ». Dans: Actes de la recherche en sciences sociales, Paris, Seuil, n 111-112, mars 1996, p. 92.

22. Sylvie Villelm, La dramaturgie implicite dans les romans de Charles-Ferdinand Ramuz (1926-1937). Thèse de doctorat, Université Aix-Marseille I - Université de Provence, 2001, p. 367-385.

23. Idem, p. 384.

24. Cité par Mervi Helkulla, «Énonciation et subjectivité dans La Première Gorgée de bière et autres plaisirs minuscules ». Dans : Poétique, Paris, Seuil, n 147, septembre 2006, p. 320.

25. Dominique Maingueneau, Éléments de linguistique pour le texte littéraire, Paris, Dunod, 1993, p. 8 .

26. Gilles Deleuze, Critique et clinique, Paris, Minuit, 1993, p. 13.

27. Charles-Ferdinand Ramuz, Derborence. Dans : Cuvres complètes, vol. 17, Genève, Slatkine, 1986, p. 187. On ne peut pas manquer de mentionner un des points qui distingue cet extrait de son analogue dans l'édition parue en 1936 à Paris chez Bernard Grasset (p. 21.). Dans l'édition de Slatkine (1986) on lit: «il y a le doigt tendu de Séraphin qui oblige l'œil s'arrêter », tandis que dans celle de Grasset (1936), on retrouve «il y a le doigt tendu de Séraphin qui oblige le regard s'arrêter ». On remarque que - d'après les différents manuscrits ramuziens - les deux versions témoignent de l'anonymité de l'instance qui pose son regard sur le doigt du personnage.

28. Ibid., p. 339.

29. Philippe Renaud, op.cit., p. 34.

30. Charles-Ferdinand Ramuz, La grande peur dans la montagne. Dans : CEuvres complètes, vol. 13, Genève, Slatkine, 1986, p. 187-188.

31. Charles-Ferdinand Ramuz, Les signes parmi nous. Dans : CEuvres complètes, vol.10, Genève, Slatkine, 1986, p. 131.

32. Cité par Kamel Skander, «La discontinuité, une esthétique de l'écriture chez Leiris. » Dans : Isabelle Chol, Poétiques de la discontinuité de 1870 à nos jours, Clermont-Ferrand, Presses Universitaires Blaise Pascal, 2004, p. 114.

33. Charles-Ferdinand Ramuz, Les signes parmi nous. Dans : CEuvres complètes, vol. 10, Genève, Slatkine, 1986, p. 83.

34. Christian Morzewski, «Regard et point de vue dans les nouvelles de Ramuz - essai d'optique ramuzienne. » Dans : Ramuz et la forme brève. (Textes présentés et réunis par Jean-Louis Pierre), La Revue des Lettres Modernes, Paris, Caen, 2003, p. 178.

35. Charles-Ferdinand Ramuz, La grande peur dans la montagne. Dans : CEuvres complètes, vol. 13, Genève, Slatkine, 1986, p. 165. 


\section{RÉSUMÉS}

L'article se propose de placer l'œuvre de l'auteur suisse romand, Charles-Ferdinand Ramuz dans une perspective de diversité esthétique. L'étude de l'instance narrative et plus particulièrement celle de l'image ramuzienne dans toute sa pluralité et son caractère discontinu permet notamment la découverte d'une esthétique basée sur une conception particulière de la langue et de l'image. Les possibles esthétiques et narratifs qui se dessinent, justifient la modernité et la complexité de l'écriture en question, ainsi que son caractère fortement cinématographique.

Der Artikel setzt sich das Ziel, das Werk des schweizer Autors Charles-Ferdinand Ramuz in einer neuen ästhetische Perspektive zu erfassen. Die Studie der narrativen Instanz und besonders die des Bildes - seine Pluralität, bzw. sein diskontinuierlicher Charakter - ermöglicht die Entdeckung einer Ästhetik, die auf einer originalen Auffassung der Sprache und des Bildes basiert. Das Mögliche in Hinsicht der Ästhetik und der Narration bekommt neue Dimensionen, die die Modernität und die Komplexität der Schreibweise von Ramuz rechtfertigen und seine kinematographischen Merkmale betonen.

INDEX

Mots-clés : cinéma, discontinuité, diversité, image, juxtaposition, langue, perception

Index chronologique : 1910-1930

Index géographique : Suisse

\section{AUTEUR}

NOÉMI KILA

Université de Pécs (Hongrie), Université d'Artois 